Notfall Rettungsmed $2021 \cdot 24: 1067-1069$ https://doi.org/10.1007/s10049-021-00943-4 Angenommen: 12. August 2021 Online publiziert: 13. September 2021 (c) Springer Medizin Verlag $\mathrm{GmbH}$, ein Teil von Springer Nature 2021

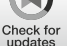

\title{
Forensischer Blick auf Rettungsmaßnahmen, Todesfeststellung und Medizinrecht
}

\author{
Notfallmedizin und Rechtsmedizin - Teil 1
}

\author{
Benjamin Ondruschka ${ }^{1}$ Florian T. Fischer ${ }^{2}$ \\ 'Institut für Rechtsmedizin, Universitätsklinikum Hamburg-Eppendorf, Hamburg, Deutschland \\ ${ }^{2}$ Institut für Rechtsmedizin, Ludwig-Maximilians-Universität München, München, Deutschland
}
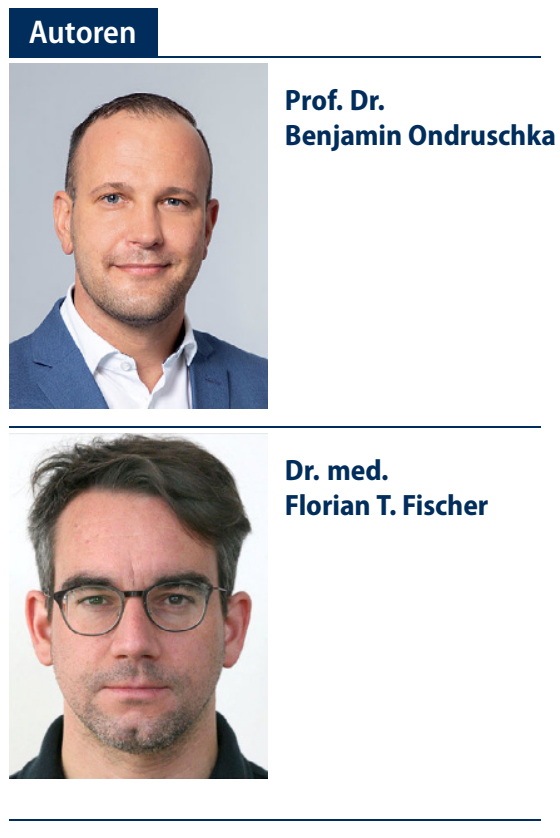

In der Ausgabe $1 / 22$ wird es den zweiten Teil des Themenhefts geben mit dem Titel Morphologie und Dokumentation von Verletzungen sowie Einsätze im Polizeigewahrsam

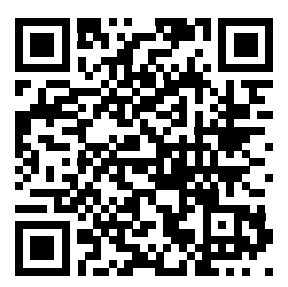

QR-Code scannen \& Beitrag online lesen
Der Umgang mit forensischen Fragen ist in der klinischen Routine sicher die Ausnahme. Sobald sich die Medizin aber „auf die Straße" begibt, tritt sie regelmäßig in Interaktion mit der öffentlichen Sicherheit und Ordnung und dann auch mit der "gerichtlichen Medizin". Die Rechtsmedizin ist das Fachgebiet der Medizin, das sich mit der Beurteilung und Anwendung medizinischer und naturwissenschaftlicher Erkenntnisse für die Rechtsprechung befasst. Ein wesentlicher Schwerpunkt sind hierbei strafrechtliche Fragestellungen. So ergeben sich zahlreiche Berührungspunkte zwischen Notfall- und Rechtsmedizin, da die beweissichere Dokumentation von Verletzungen, die Todesfeststellung und Leichenschau, die Interaktion mit der Polizei und zahlreiche medizinrechtliche Aspekte in gewisser Weise "grenznah" lokalisiert sind. Besonders deutlich wird dies, da das Notfallteam häufig der erste Kontakt ist, den Geschädigte einer Straftat haben, sei es als Verletzte oder gar Getötete, aber auch als Verstorbene mit unklarer Vorgeschichte. Das Notfallteam ist aber auch, vielleicht der Natur der Sache geschuldet, häufig selbst im Fokus forensischer Begutachtung, sind die Umstände des Einzelfalls doch öfter unklar als im stationären Setting und die Bedingungen schwierig(er) - mit der Konsequenz, dass von Angehörigen oder Beteiligten öfter Vorwürfe erhoben werden, die einer medikolegalen Aufklärung bedürfen.
Beim Eintritt des Todes im Zusammenhang mit einer notfallmedizinischen Behandlung, vor allem aus einer akuten lebensbedrohlichen Situation zunächst unbekannter Ursache, steht für die leichenschauhaltenden Ärzte häufig eine ungeklärte oder nicht natürliche Todesart im Raum. Die Leichenschau stellt hier die Weichen und leitet polizeiliche Basisarbeit ein. Schröder et al. zeigen die Grundlagen auf, die für den rechtssicheren Umgang mit der Todesfeststellung und der Leichenschau unabdingbar sind.

\section{I) Zwischen Notfall- und}

Rechtsmedizin gibt es zahlreiche

Berührungspunkte

Auch wenn nicht jeder Patient erfolgreich behandelt werden kann, ist es doch ärztliche Aufgabe, daraus zu lernen. Die Notfallmedizin kann aus den Erkenntnissen der Rechtsmedizin und postmortalen Forschung einen erheblichen Nutzen ziehen, da eine Verbesserung insbesondere durch eine autoptische Aufarbeitung der Qualität von Diagnosen und Therapien bei Verstorbenen erreicht werden kann. Rössler et al. zeigen dies an der Anwendung der Beckenschlinge auf.

Betrachtet man die Entwicklung im Rettungsdienst, so ist die immer besser werdende Ausbildung und Spezialisierung, vom Rettungssanitäter über den Rettungsassistenten zum Notfallsanitäter, sehr begrüßenswert. Parallel wachsen 
aber auch die Anforderungen in der Notfallmedizin aufgrund der neuen Kompetenzen, mit entsprechendem Mehr an Verantwortung und auch Haftung. Die Notfallmedizin findet sich immer häufiger als Gegenstand rechtsmedizinischer Betrachtungen wieder. Abgesehen von klassischen Behandlungsfehlern aktiver oder auch passiver Natur werden die Situationen vor dem Hintergrund der aktuellen Rechtsprechung zum Umgang mit Patientenverfügungen, zum selbstbestimmten Sterben und der Ausübung der "Heilkunde" zunehmend komplexer und damit auch fehleranfälliger. Hier soll der Beitrag von Dettmeyer et al. sensibilisieren und das Problembewusstsein aller Kollegen schärfen.

Betrachtet man die Erfahrungen, die wir an dieser Grenze zwischen Notfallund Rechtsmedizin machen konnten, so kann man eine enge interdisziplinäre $\mathrm{Zu}$ sammenarbeit zum beidseitigen Nutzen nur empfehlen. Beide Fächer profitieren im Sinne der Patientenversorgung und Rechtssicherheit erheblich voneinander.

\section{B. Ondruschka \\ F.T. Fischer}

\section{Korrespondenzadresse}

\section{Prof. Dr. Benjamin Ondruschka}

Institut für Rechtsmedizin, Universitätsklinikum Hamburg-Eppendorf

Butenfeld 34, 22529 Hamburg, Deutschland

b.ondruschka@uke.de

\section{Dr. med. Florian T. Fischer}

Institut für Rechtsmedizin, Ludwig-

Maximilians-Universität München

Nußbaumstraße 26, 80336 München,

Deutschland

florian.fischer@med.uni-muenchen.de

Interessenkonflikt. B. Ondruschka und F.T. Fischer geben an, dass kein Interessenkonflikt besteht.

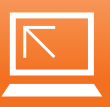

\section{Dank an die Gutachter*innen}

Für die Qualität und Objektivität der Beiträge sind neben den engagierten Autorinnen und Autoren auch die vielen qualifizierten Gutachterinnen und Gutachter maßgeblich, die im Rahmen des Peer-Review-Prozesses die Manuskripte inhaltlich-wissenschaftlich prüfen und Empfehlungen zur konkreten Verbesserung äußern. Allen, die im vergangenen Jahr Manuskripte für diese Zeitschrift begutachtet haben, danken wir herzlich für die konstruktive und gewissenhafte Arbeit.

Die Redaktion

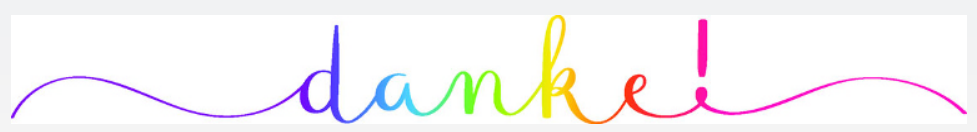

() treenabeena / stock.adobe.com 
Hier steht eine Anzeige.

黑 Springer 